\title{
Contracting the Socle in Rings of Continuous Functions
}

\author{
Themba Dube $(*)$
}

ABSTRACT - Not every ring homomorphism contracts the socle of its codomain to an ideal contained in the socle of its domain. Rarer still does a homomorphism contract the socle to the socle. We find conditions on a frame homomorphism that ensure that the induced ring homomorphism contracts the socle of the codomain to an ideal contained in the socle of the domain. The surjective among these frame homomorphisms induce ring homomorphisms that contract the socle to the socle. These homomorphisms characterize $P$-frames as those $L$ for which every frame homomorphism with $L$ as domain is of this kind.

\section{Introduction.}

The socle of a ring, it will be recalled, is the ideal generated by the minimal ideals of the ring. In [14] the authors show that, for a completely regular Hausdorff space $X$, the socle of the ring $C(X)$ is the ideal consisting of all functions which are zero everywhere except on a finite number of points. Their method consists of first describing minimal ideals of $C(X)$.

Because our main aim is to find conditions on a frame homomorphism $h: L \rightarrow M$ which ensure that the induced ring homomorphism $\mathcal{R} h: \mathcal{R} L \rightarrow$ $\rightarrow \mathcal{R} M$ contracts the socle to an ideal contained in the socle, we take a different approach in characterizing the socle of the ring $\mathcal{R} L$ of real-valued continuous functions on a completely regular frame $L$. What we do is first give a description of annihilators of elements of $\mathcal{R} L$ (Lemma 3.1), and then use the following characterization of the socle

$$
x \in \operatorname{Soc} A \Leftrightarrow \operatorname{Ann} x \text { is an intersection of finitely many maximal ideals, }
$$

to show that the socle of $\mathcal{R} L$ is the ideal consisting of functions whose cozero elements are finite joins of atoms of $L$ (Proposition 3.5). The spatial result is

(*) Indirizzo dell'A.: Department of Mathematical Sciences, University of South Africa, P. O. Box 392, 0003 Unisa, South Africa.

E-mail: dubeta@unisa.ac.za 
then obtained as a corollary. We point out how else Proposition 3.5 could have been proved if the sole aim had only been to do that.

Recall [9] that a ring homomorphism $\phi: A \rightarrow B$ is said to be exoteric if whenever a pair of finitely generated ideals of $A$ have the same annihilator, then their images under $\phi$ have the same annihilator. An ideal is then said to be exoteric if it is the kernel of some exoteric homomorphism. We show, using a convenient characterization in [9], that Soc $\mathcal{R} L$ is exoteric (Proposition 3.8). In the case that $L$ has a finite number of atoms, we actually produce a ring homomorphism with domain $\mathcal{R} L$ whose kernel is Soc $\mathcal{R} L$.

Every frame homomorphism $h: L \rightarrow M$ induces a ring homomorphism $\mathcal{R} h: \mathcal{R} L \rightarrow \mathcal{R} M$ which sends an element $\alpha$ of $\mathcal{R} L$ to the composite $h \circ \alpha$. In the last section we identify a certain class of frame homomorphisms (we call them W-maps) $h: L \rightarrow M$ for which $(\mathcal{R} h)^{-1}[\operatorname{Soc} \mathcal{R} M] \subseteq \operatorname{Soc} \mathcal{R} L$ (Proposition 4.6). If $h$ is a dense onto W-map, then, in fact, $(\mathcal{R} h)^{-1}[\operatorname{Soc} \mathcal{R} M]=\operatorname{Soc} \mathcal{R} L$. These homomorphisms are defined in terms of the frame version of the Stone extension of a continuous map. An internal characterization free of the Stone-Čech compactification is presented (Lemma 4.3). A ring-theoretic characterization is that $h$ is a W-map if and only if $\mathcal{R} h$ contracts every maximal ideal to a maximal ideal (Proposition 4.9). Rather unexpectedly, Wmaps characterize $P$-frames (i.e. frame counterparts of $P$-spaces) as those $L$ for which every frame homomorphism with $L$ as domain is of this kind (Proposition 4.4).

An example of a dense onto frame homomorphism $h$ for which $\mathcal{R} h$ is not an isomorphism but $(\mathcal{R} h)^{-1}[\operatorname{Soc} \mathcal{R} M]=\operatorname{Soc} \mathcal{R} L$ is given (Example 4.5). Also, an example of a dense onto frame homomorphism $h: L \rightarrow M$ which is not a W-map but for which $(\mathcal{R} h)^{-1}[\operatorname{Soc} \mathcal{R} M]=\operatorname{Soc} \mathcal{R} L$ is given (Example 4.8).

\section{Notation and a few reminders.}

For a general theory of frames we refer to [13]. Here we collect a few facts that will be relevant for our discussion. A frame is a complete lattice $L$ in which the distributive law

$$
a \wedge \bigvee S=\bigvee\{a \wedge x \mid x \in S\}
$$

holds for all $a \in L$ and $S \subseteq L$. We denote the top element and the bottom element of $L$ by 1 and 0 respectively. The frame of open subsets of a topological space $X$ is denoted by $\subseteq X$. All spaces are assumed to be Tychonoff, that is, completely regular and Hausdorff. 
An element $a$ of $L$ is rather below (or well below) an element $b$, written $a \prec b$, if there is an element $s$ such that $a \wedge s=0$ and $s \vee b=1$. On the other hand, $a$ is completely below $b$, written $a \prec \prec b$, if there are elements $\left(x_{r}\right)$ indexed by the rational numbers in $\mathrm{Q} \cap[0,1]$ such that $a=x_{0}, x_{1}=b$ and $x_{r} \prec x_{s}$ for $r<s$. $L$ is then said to be regular if $a=\bigvee\{x \in L \mid x \prec a\}$ for each $a \in L$, and completely regular if $a=\bigvee\{x \in L \mid x \prec \prec a\}$ for each $a \in L$. Although we shall assume all frames considered here to be completely regular, there are instances where we state minor results for regular frames, which then hold for completely regular frames since every completely regular frame is regular.

The pseudocomplement of an element $a$ is the element $a^{*}=$ $=\bigvee\{x \in L \mid x \wedge a=0\}$. An element $a$ is said to be:

(1) complemented if $a \vee a^{*}=1$,

(2) dense if $a^{*}=0$,

(3) an atom if, for any $x \in L, 0 \leq x \leq a$ implies $x=0$ or $x=a$,

(4) a point (or a prime element) if $a<1$ and $x \wedge y \leq a$ implies $x \leq a$ or $y \leq a$.

A frame is atomic if below every nonzero element there is an atom. The meet of finitely many dense elements is dense. If $c_{1}, \ldots, c_{m}$ are finitely many complemented elements, then $c_{1} \wedge \cdots \wedge c_{m}$ is complemented with $\left(c_{1} \wedge \cdots \wedge c_{m}\right)^{*}=c_{1}^{*} \vee \cdots \vee c_{m}^{*}$. The points of any regular frame are precisely those elements which are maximal below the top (see, for instance, [3, page 12]). Modulo the Axiom of Choice (in the form of Zorn's Lemma), any compact frame has enough points in the sense that every element is the meet of points above it; with the convention that the empty meet is the top element. It is for this reason, among others, that we embrace AC throughout.

A frame homomorphism is a map between frames which preserves finite meets and arbitrary joins. By a quotient map we mean an onto frame homomorphism. For any $a \in L, \downarrow a$ denotes the set $\{x \in L \mid x \leq a\}$, which is a frame in its own right. We then have the open quotient map $L \rightarrow \downarrow a$ sending any $x \in L$ to $a \wedge x$. A frame homomorphism is called dense if it maps only the bottom element to the bottom element. If $h$ is a dense onto frame homomorphism, then $h\left(a^{*}\right)=h(a)^{*}$ for all $a$. Associated with a frame homomorphism $h: L \rightarrow M$ is its right adjoint $h_{*}: M \rightarrow L$ given by

$$
h_{*}(a)=\bigvee\{x \in L \mid h(x) \leq a\} .
$$

Recall that $h_{*}$ preserves meets, and hence $h_{*}(a) \leq h_{*}(b)$ whenever $a \leq b$. 
An ideal $J$ of $L$ is completely regular if for each $x \in J$ there exists $y \in J$ such that $x \prec \prec y$. For a completely regular frame $L$, the frame of its completely regular ideals is denoted by $\beta L$. For any $a \in L, \downarrow a \in \beta L$ if and only if $a$ is complemented. The join map $\beta L \rightarrow L$ is a dense onto frame homomorphism, and $\beta L$ (together with the join map) is referred to as the Stone-Cech compactification of $L$. We denote the right adjoint of the join map $\beta L \rightarrow L$ by $r_{L}$ (dropping the subscript when it is unnecessary), and recall that for any $a \in L$ and $I \in \beta L$ :

(1) $r(a)=\{x \in L \mid x \prec \prec a\}$,

(2) $r\left(a^{*}\right)=r(a)^{*}$,

(3) $I^{*}=r\left((\bigvee I)^{*}\right)$.

Regarding the frame of reals $\mathcal{L}(\mathbb{R})$ and the ring $\mathcal{R} L$ of continuous real-valued functions on $L$; we use the notation of [2]. It will be recalled that $\mathcal{R} L$ is the collection of frame homomorphisms from $\mathcal{L}(\mathbb{R})$ into $L$. See also [1] for some properties of the ring $\mathcal{R} L$. The cozero map coz: $\mathcal{R} L \rightarrow L$ is given by

$$
\operatorname{coz} \varphi=\bigvee\{\varphi(p, 0) \vee \varphi(0, q) \mid p, q \in \mathbb{Q}\}=\varphi((-\infty, 0) \vee(0, \infty)) .
$$

Some of its properties that we shall frequently use include the following:

$(1 \operatorname{coz} \gamma \delta=\operatorname{coz} \gamma \wedge \operatorname{coz} \delta$,

(2) $\operatorname{coz}(\gamma+\delta) \leq \operatorname{coz} \gamma \vee \operatorname{coz} \delta$,

(3) $\operatorname{coz}(\gamma+\delta)=\operatorname{coz} \gamma \vee \operatorname{coz} \delta$ if $\gamma, \delta \geq \mathbf{0}$,

(4) $\operatorname{coz} \varphi=0$ iff $\varphi=\mathbf{0}$.

A frame homomorphism $h: L \rightarrow M$ induces a ring homomorphism $\mathcal{R} h: \mathcal{R} L \rightarrow \mathcal{R} M$ which sends an element $\alpha$ of $\mathcal{R} L$ to $h \circ \alpha$. Furthermore, $\operatorname{coz}(h \circ \alpha)=(h \circ \alpha)((-\infty, 0) \vee(0, \infty))=h(\alpha((-\infty, 0) \vee(0, \infty)))=h(\operatorname{coz} \alpha)$

A cozero element of $L$ is an element of the form $\operatorname{coz} \varphi$ for some $\varphi \in \mathcal{R} L$. The cozero part of $L$, denoted $\operatorname{Coz} L$, is the regular sub- $\sigma$-frame consisting of all the cozero elements of $L$. A frame is completely regular if and only if it is generated by its cozero part, in the sense that every element is the join of cozero elements below it. General properties of cozero elements and cozero parts of frames can be found in [5].

Lastly, by "ring" we mean a commutative ring with identity. The annihilator of subset $S$ is denoted by Ann $S$ and that of an element $a$ by Ann $a$. $\mathrm{By} \mathrm{Ann}{ }^{2}$ we mean the annihilator of the annihilator. An ideal generated by elements $a_{1}, \ldots, a_{m}$ is written as $\left\langle a_{1}, \ldots, a_{m}\right\rangle$. 


\section{Characterizing the socle of $\mathcal{R} L$.}

We start by recalling some relevant facts about $\boldsymbol{M}$-ideals of $\mathcal{R} L$ that were introduced in [6]. For any $I \in \beta L$, the ideal $\boldsymbol{M}^{I}$ of $\mathcal{R} L$ is defined by

$$
\boldsymbol{M}^{I}=\{\varphi \in \mathcal{R} L \mid r(\operatorname{coz} \varphi) \subseteq I\} .
$$

Clearly, for any $I, J \in \beta L, \boldsymbol{M}^{I}=\boldsymbol{M}^{J}$ implies $I=J$, and $\boldsymbol{M}^{I} \cap \boldsymbol{M}^{J}=\boldsymbol{M}^{I \wedge J}$. It is proved in [6], Proposition 5.1, that an ideal of $\mathcal{R} L$ is maximal if and only if it is of the form $\boldsymbol{M}^{I}$ for some point $I$ of $\beta L$.

We require a series of lemmas to be able to prove the main result in this section. The first of these gives a description of annihilators of elements of $\mathcal{R} L$. We prove a more general result than is needed.

Lemma 3.1. Let $S \subseteq \mathcal{R} L$ and put $a=\bigvee\{\operatorname{coz} \alpha \mid \alpha \in S\}$. Then Ann $S=M^{r\left(a^{*}\right)}$. In particular, for any $\varphi \in \mathcal{R} L$, Ann $\varphi=\boldsymbol{M}^{r\left((\operatorname{coz} \varphi)^{*}\right)}$. Actually, annihilator ideals of $\mathcal{R} L$ are precisely the ideals $\boldsymbol{M}^{r\left(b^{*}\right)}$, for $b \in L$.

Proof. For any $\varphi \in \mathcal{R} L$ we have

$$
\begin{aligned}
\varphi \in \operatorname{Ann} S & \Leftrightarrow \varphi \alpha=\mathbf{0} \text { for each } \alpha \in S \\
& \Leftrightarrow \operatorname{coz} \varphi \wedge \operatorname{coz} \alpha=0 \text { for each } \alpha \in S \\
& \Leftrightarrow \operatorname{coz} \varphi \wedge a=0 \\
& \Leftrightarrow \operatorname{coz} \varphi \leq a^{*} \\
& \Leftrightarrow r(\operatorname{coz} \varphi) \subseteq r\left(a^{*}\right) \\
& \Leftrightarrow \varphi \in \boldsymbol{M}^{r\left(a^{*}\right)},
\end{aligned}
$$

which proves the first part. Now, to prove the second part, let $b \in L$. Put $B=\{\alpha \in \mathcal{R} L \mid \operatorname{coz} \alpha \leq b\}$. Then $b=\bigvee\{\operatorname{coz} \alpha \mid \alpha \in B\}$ by complete regularity. So, by the first part, $\boldsymbol{M}^{r\left(b^{*}\right)}=\operatorname{Ann} B$.

REMARK 3.2. (1) Recall that an ideal of a ring is said to be essential if it has nonzero intersection with every nonzero ideal. For reduced rings this is equivalent to saying the annihilator of the ideal is the zero ideal. The singular ideal of a ring $A$ is the ideal

$$
Z(A)=\{a \in A \mid \text { Ann } a \text { is essential }\} .
$$

Since an ideal of a reduced commutative ring is essential if and only if its annihilator is zero, we see from the lemma that $Z(\mathcal{R} L)=\{\boldsymbol{0}\}$, since $\varphi \in Z(\mathcal{R} L)$ iff Ann $\varphi$ is essential, iff $\operatorname{Ann} \boldsymbol{M}^{r\left((\operatorname{coz} \varphi)^{*}\right)}=\{\boldsymbol{0}\}$, iff $\boldsymbol{M}^{r\left((\operatorname{coz} \varphi)^{* *}\right)}=\{\mathbf{0}\}$, iff $\operatorname{coz} \varphi=0$, iff $\varphi=\mathbf{0}$. 
(2) In [11] a ring $A$ is said to have the countable annihilator condition (abbreviated cac) if given any countable set $\left\{a_{n} \mid n \in \mathbb{N}\right\} \subseteq A$, there exists $a \in A$ such that $\bigcap_{n}$ Ann $a_{n}=$ Ann $a . \mathcal{R} L$ has the cac, for if $\left(\gamma_{n}\right)$ is a sequence in $\mathcal{R} L$ and $\gamma$ is an element of $\mathcal{R} L$ such that $\operatorname{coz} \gamma=\bigvee \operatorname{coz} \gamma_{n}$, then, using Lemma 3.1, one shows that $\bigcap_{n} \operatorname{Ann} \gamma_{n}=\operatorname{Ann} \gamma$.

Next, a quick lemma concerning pseudocomplements. The proof is straightforward and therefore not included.

Lemma 3.3. Suppose $a=c \wedge d$ with $d$ dense. Then $a^{*}=c^{*}$.

For the following lemma, note that if $a$ is an atom in a regular frame, then $a$ is complemented. Indeed, by regularity, there exists $0 \neq x \prec a$. But then, in light of $a$ being an atom, $x=a$, which implies $a \prec a$, whence $a^{*} \vee a=1$.

LEMMA 3.4. The following relations between atoms of $L$ and points of BL hold:

(a) If I is a point of $\beta L$, then $(\bigvee I)^{*}$ is the bottom element or an atom of $L$.

(b) If a is an atom of $L$, then $r\left(a^{*}\right)$ is a point of $\beta L$.

Proof. (a) Note first that every point of any frame is either dense or complemented. If $I$ is dense, then $\bigvee I$ is dense, and hence $(\bigvee I)^{*}=0$. Now suppose $I$ is complemented, and consider any $x \in L$ with $0<x \leq(\bigvee I)^{*}$. Then $x^{*} \neq 1$, and hence $r\left(x^{*}\right) \neq 1_{\beta L}$. Since $I \leq I^{* *}=r\left((\bigvee I)^{* *}\right) \leq r\left(x^{*}\right) \neq 1_{\beta L}$, it follows from $I$ being a point that $I=r\left(x^{*}\right)$. Consequently, $\bigvee I=x^{*}$, and hence $x^{* *}=(\bigvee I)^{*}$. Now let $0<c \leq(\bigvee I)^{*}$. By complete regularity, there exists $0 \neq x \prec \prec c$. Then $x^{*} \vee c=1$. But $x^{* *}=c^{* *}$ since, by what we have shown so far, each of these elements equals $(\bigvee I)^{*}$. So $x^{*}=c^{*}$, and hence $c \vee c^{*}=1$, which implies $c=c^{* *}=(\bigvee I)^{*}$. Therefore $(\bigvee I)^{*}$ is an atom.

(b) Because the right adjoint of a frame homomorphism preserves points, it suffices to show that $a^{*}$ is a point of $L$. Let $s \in L$ such that $a^{*}<s \leq 1$. Then $s^{*} \leq a^{* *}=a$. But $s^{*} \neq a$, so $s^{*}=0$, implying that $s=1$.

In the following proof we shall need to take cognizance of the fact that if $c_{1}, \ldots, c_{m}$ are finitely many atoms and $a \leq c_{1} \vee \cdots \vee c_{m}$, then $a$ is a join of finitely many atoms. For, $a=\left(a \wedge c_{1}\right) \vee \cdots \vee\left(a \wedge c_{m}\right)$, and each $a \wedge c_{i}$ is either zero or $c_{i}$. 
Proposition 3.5. Soc $\mathcal{R} L=\{\varphi \in \mathcal{R} L \mid \operatorname{coz} \varphi$ is a join of finitely many atoms\}.

Proof. Let $\varphi \in \operatorname{Soc} \mathcal{R} L$. Take finitely many points $I_{1}, \ldots, I_{k}$ of $\beta L$ such that

$$
\text { Ann } \varphi=\boldsymbol{M}^{I_{1}} \cap \cdots \cap \boldsymbol{M}^{I_{k}} .
$$

For brevity, write $\operatorname{coz} \varphi=a$. Therefore, by Lemma 3.1, $\boldsymbol{M}^{r\left(a^{*}\right)}=\boldsymbol{M}^{I_{1} \wedge \cdots \wedge I_{k}}$, which implies $r\left(a^{*}\right)=I_{1} \wedge \cdots \wedge I_{k}$. We claim that $a \leq\left(\bigvee I_{1}\right)^{*} \vee \cdots \vee\left(\bigvee I_{k}\right)^{*}$. Let $I$ denote the meet of all the complemented elements of $\left\{I_{1}, \ldots, I_{k}\right\}$ and $J$ the meet of the dense ones. Then $I$ is complemented and $J$ dense. Since $r\left(a^{*}\right)=I \wedge J$, Lemma 3.3 yields $r\left(a^{* *}\right)=I^{*}=I_{i_{1}}^{*} \vee \cdots \vee I_{i_{m}}^{*}$ for some indices $i_{1}, \ldots, i_{m}$. Taking joins yields

$$
a \leq a^{* *}=\bigvee I_{i_{1}}^{*} \vee \cdots \vee \bigvee I_{i_{m}}^{*}=\left(\bigvee I_{i_{1}}\right)^{*} \vee \cdots \vee\left(\bigvee I_{i_{m}}\right)^{*},
$$

since the join map, being dense and onto, commutes with pseudocomplementation; that is, $(\bigvee J)^{*}=\bigvee J^{*}$ for each $J \in \beta L$. It follows from Lemma 3.4(a) that $a$ is a join of finitely many atoms; proving the inclusion $\subseteq$.

Conversely, suppose $\varphi$ is an element of $\mathcal{R} L$ such that $\operatorname{coz} \varphi=$ $=a_{1} \vee \cdots \vee a_{k}$, where the $a_{i}$ are finitely many atoms. Then $(\operatorname{coz} \varphi)^{*}=$ $=a_{1}^{*} \wedge \cdots \wedge a_{k}^{*}$, and hence

$$
r\left((\operatorname{coz} \varphi)^{*}\right)=r\left(a_{1}^{*}\right) \wedge \cdots \wedge r\left(a_{k}^{*}\right)
$$

since $r$ preserves meets. Thus, by Lemma 3.1

$$
\text { Ann } \varphi=\boldsymbol{M}^{r\left((\operatorname{coz} \varphi)^{*}\right)}=\boldsymbol{M}^{r\left(a_{i}^{*}\right)} \cap \cdots \cap \boldsymbol{M}^{r\left(a_{k}^{*}\right)} .
$$

Now, by Lemma 3.4(b), $r\left(a_{i}^{*}\right)$ is a point of $\beta L$ for each $i$, so $\varphi \in \operatorname{Soc} \mathcal{R} L$.

CoRollaRY 3.6. If $h: L \rightarrow M$ is onto, then $(\mathcal{R} h)[\operatorname{Soc} \mathcal{R} L] \subseteq \operatorname{Soc} \mathcal{R} M$.

Proof. If Soc $\mathcal{R} L$ is zero, there is nothing to prove. So suppose otherwise and let $\varphi \in \operatorname{Soc} \mathcal{R} L$. By Proposition 3.5, there are finitely many atoms $a_{1}, \ldots, a_{m}$ in $L$ such that $\operatorname{coz} \varphi=a_{1} \vee \cdots \vee a_{m}$. Therefore

$$
\operatorname{coz}(h \circ \varphi)=h(\operatorname{coz} \varphi)=h\left(a_{1}\right) \vee \cdots \vee h\left(a_{m}\right) .
$$

We claim that for any atom $a$ of $L, h(a)$ is an atom of $M$. Let $y \in M$ be such that $y<h(a)$. We must show that $y=0$. Since $h$ is onto, there exists $x \in L$ such that $h(x)=y$. By regularity, $x=\bigvee\{s \in L \mid s \prec x\}$. Take any $t \prec x$ in $L$. Then $t^{*} \vee x=1$. Therefore $a=\left(a \wedge t^{*}\right) \vee(a \wedge x)$. Since $a$ is an atom, 
$a \wedge x=0$ or $a \wedge x=a$. The latter is not possible, lest we have $a \leq x$, whence $h(a) \leq h(x)=y$, contrary to the fact that $y<h(a)$. Thus, $a \wedge x=0$, which implies $0=h(a) \wedge h(x)=h(a) \wedge y=y$, as required. Therefore $\operatorname{coz}(h \circ \varphi)$ is a join of finitely many atoms, and hence $(\mathcal{R} h)(\varphi)=h \circ \varphi \in \operatorname{Soc} \mathcal{R} M$.

The spatial result mentioned in the introduction is a corollary of Proposition 3.5. To see this, note first that, for any $\mathrm{T}_{1}$-space $X$, the atoms of $\subseteq X$ are precisely the open singletons. If $\phi: A \rightarrow B$ is a ring isomorphism, then $\phi[\operatorname{Soc} A]=\operatorname{Soc} B$. For any topological space $X$, the ring isomorphism $C(X) \rightarrow \mathcal{R}(\leqq X)$ taking $f \in C(X)$ to $\varphi \in \mathcal{R} L$ such that, for all $p, q \in \mathrm{Q}$,

$$
\varphi(p, q)=f^{-1}[\{x \in \mathbb{R} \mid p<x<q\}],
$$

has the property that

$$
\operatorname{coz} \varphi=\{x \in X \mid f(x) \neq 0\},
$$

the cozero-set of $f$ (see [2]). Therefore, for any $f \in C(X), f \in \operatorname{Soc} C(X)$ if and only if the cozero set of $f$ is a join (in $\bigcirc X$ ) of finitely many atoms. Thus, $f \in \operatorname{Soc} C(X)$ if and only if it is a union of finitely many open singletons; which is precisely the spatial result in question.

Remark 3.7. By Lemma 3.1 and Proposition 3.5, Ann $(\operatorname{Soc} \mathcal{R} L)=$ $=\boldsymbol{M}^{r\left(a^{*}\right)}$ where $a$ denotes the join of all atoms of $L$. Thus, Soc $\mathcal{R} L$ is essential iff $a^{*}=0$, iff $a$ is dense, which, in turn, holds iff every nonzero element of $L$ has a nonzero meet with some atom, equivalently, $L$ is atomic.

A ring homomorphism $\phi: A \rightarrow B$ is called exoteric [9] if for all pairs $(I, J)$ of finitely generated ideals of $A$, Ann $I=\operatorname{Ann} J$ implies Ann $\phi[I]=$ $=$ Ann $\phi[J]$. An exoteric ideal is an ideal which is a kernel of an exoteric homomorphism. It is shown in [9], Theorem 2.6, that an ideal $I$ of a ring $A$ is exoteric if and only if for any finite set $\left\{a_{1}, \ldots, a_{m}\right\} \subseteq I$, $\operatorname{Ann}^{2}\left(\sum_{i} \operatorname{Ann}^{2} a_{i}\right) \subseteq I$. We show that Soc $\mathcal{R} L$ is exoteric. We need two preliminary results from [7], namely Lemma 2.1 and Proposition 2.2 which, respectively, state:

If $\alpha, \beta \in \mathcal{R} L$ and $\operatorname{coz} \alpha \prec \prec \operatorname{coz} \beta$, then $\alpha$ is a multiple of $\beta$, and

The map $\eta \mapsto \operatorname{coz} \eta$ is an isomorphism between the Boolean algebra of idempotents of $\mathcal{R} L$ and the Boolean algebra of complemented elements of $L$. 
Proposition 3.8. Soc $\mathcal{R} L$ is an exoteric ideal.

Proof. Let $\alpha_{1}, \ldots, \alpha_{m}$ be finitely many elements of Soc $\mathcal{R} L$. For each $i \in\{1, \ldots, m\}$ find atoms $a_{i 1}, \ldots, a_{i n_{i}}$ such that $\operatorname{coz} \alpha_{i}=a_{i 1} \vee \cdots \vee a_{i n_{i}}$. Write the sequence

$$
a_{11}, \ldots, a_{1 n_{1}}, \ldots, a_{m 1}, \ldots a_{m n_{m}}
$$

as $a_{1}, \ldots, a_{k}$. Find idempotents $\eta_{1}, \ldots, \eta_{k}$ in $\mathcal{R} L$ such that $a_{j}=\operatorname{coz} \eta_{j}$ for each $j \in\{1, \ldots, k\}$. Now, since the join of finitely many complemented elements is complemented, and since idempotents are nonnegative in the $f$-ring $\mathcal{R} L$, for each $i \in\{1, \ldots, m\}$ we have

$$
\operatorname{coz} \alpha_{i} \leq \operatorname{coz} \eta_{1} \vee \cdots \vee \operatorname{coz} \eta_{k} \prec \prec \operatorname{coz} \eta_{1} \vee \cdots \vee \operatorname{coz} \eta_{k}=\operatorname{coz}\left(\eta_{1}+\cdots+\eta_{k}\right) .
$$

Consequently, $\alpha_{i}$ is a multiple of $\eta_{1}+\cdots+\eta_{k}$, and hence $\alpha_{i} \in\left\langle\eta_{1}, \ldots, \eta_{m}\right\rangle \subseteq$ $\subseteq$ Soc $\mathcal{R} L$. Therefore

$$
\begin{aligned}
\operatorname{Ann}^{2} \alpha_{i} & \subseteq \operatorname{Ann}^{2}\left\langle\eta_{1}, \ldots, \eta_{k}\right\rangle \\
& =\operatorname{Ann} \boldsymbol{M}^{r\left(a_{1} \vee \cdots \vee a_{k}\right)^{*}} \quad \text { by Lemma } 3.1 \\
& =\boldsymbol{M}^{r\left(a_{1} \vee \cdots \vee a_{k}\right)^{* *}} \\
& =\boldsymbol{M}^{r\left(a_{1} \vee \cdots \vee a_{k}\right)},
\end{aligned}
$$

the last equality since $a_{1} \vee \cdots \vee a_{k}$ is complemented. But $\boldsymbol{M}^{r\left(a_{1} \vee \cdots \vee a_{k}\right)} \subseteq$ $\left\langle\eta_{1}, \ldots, \eta_{m}\right\rangle$, for if $\varphi \in \boldsymbol{M}^{r\left(a_{1} \vee \cdots \vee a_{k}\right)}$, then $r(\operatorname{coz} \varphi) \leq r\left(a_{1} \vee \cdots \vee a_{k}\right)$, so that

$$
\operatorname{coz} \varphi \leq \operatorname{coz}\left(\eta_{1}+\cdots+\eta_{k}\right) \prec \prec \operatorname{coz}\left(\eta_{1}+\cdots+\eta_{k}\right) .
$$

It therefore follows that $\sum_{i}\left(\mathrm{Ann}^{2} \alpha_{i}\right) \subseteq\left\langle\eta_{1}, \ldots, \eta_{m}\right\rangle$, whence

$$
\operatorname{Ann}^{2} \sum_{i}\left(\operatorname{Ann}^{2} \alpha_{i}\right) \subseteq\left\langle\eta_{1}, \ldots, \eta_{m}\right\rangle \subseteq \operatorname{Soc} \mathcal{R} L
$$

as required.

In the case that a frame has only finitely many atoms, we can actually identify a ring homomorphism whose kernel is the socle in question. We need a bit of background.

Recall [1] that an onto frame homomorphism $h: L \rightarrow M$ is called a $C$ quotient map precisely when the ring homomorphism $\mathcal{R} h$ is onto. Also, $h$ is said to be coz-onto if for any $c \in \operatorname{Coz} M$ there exists $d \in \operatorname{Coz} L$ such that $h(d)=c$. Lastly, $h$ is almost coz-codense if for any $c \in \operatorname{Coz} L$ with $h(c)=1$, there exists $d \in \operatorname{Coz} L$ such that $c \vee d=1$ and $h(d)=0$. It is shown in [1], 
Theorem 7.2.7, that an onto homomorphism is a $C$-quotient map if and only if it is coz-onto and almost coz-codense. Next, recall that $h$ is nearly open if it commutes with pseudocomplements. In particular, if $a \in L$ and $g: L \rightarrow \downarrow a$ is the open quotient map $x \mapsto x \wedge a$, then $g$ is nearly open. Dense onto homomorphisms are also nearly open.

Now if $c$ is a complemented element of $L$, then the open quotient map $g: L \rightarrow \downarrow a$ is a $C$-quotient map. That it is coz-onto follows from [1], Corollary 3.2.11, since $c$ is a cozero element. To see that it is almost coz-codense, let $u \in \operatorname{Coz} L$ such that $g(u)=1_{\downarrow c}$. Then $u \wedge c=c$, so that $c \leq u$. Now $c^{*}$ is a cozero element of $L$ such that $u \vee c^{*}=1$ (as $\left.c \vee c^{*}=1\right)$ and $g\left(c^{*}\right)=0$.

LEMma 3.9. Let $h: L \rightarrow M$ be a nearly open frame homomorphism. Then $\mathcal{R} h$ is exoteric.

Proof. Let $I=\left\langle\alpha_{1}, \ldots, \alpha_{m}\right\rangle$ and $J=\left\langle\beta_{1}, \ldots, \beta_{k}\right\rangle$ be a pair of finitely generated ideals of $\mathcal{R} L$ such that $\operatorname{Ann} I=\operatorname{Ann} J$. For brevity, write $\operatorname{coz} \alpha_{i}=a_{i}, \quad \operatorname{coz} \beta_{i}=b_{i}, \quad a_{1} \vee \cdots \vee a_{m}=a$ and $b_{1} \vee \cdots \vee b_{k}=b$. Then, $\bigvee\{\operatorname{coz} \varphi \mid \varphi \in I\}=a$, and $\bigvee\{\operatorname{coz} \varphi \mid \varphi \in J\}=b$. Thus, by Lemma 3.1,

$$
\boldsymbol{M}^{r_{L}\left(a^{*}\right)}=\operatorname{Ann} I=\operatorname{Ann} J=\boldsymbol{M}^{r_{L}\left(b^{*}\right)},
$$

so that $a^{*}=b^{*}$. Now, a simple calculation shows that

$$
\bigvee\{\operatorname{coz} \tau \mid \tau \in(\mathcal{R} h)[I]\}=h(a),
$$

and

$$
\bigvee\{\operatorname{coz} \tau \mid \tau \in(\mathcal{R} h)[J]\}=h(b) .
$$

Thus,

$$
\operatorname{Ann}(\mathcal{R} h)[I]=\boldsymbol{M}^{r_{M}\left(h(a)^{*}\right)} \quad \text { and } \quad \operatorname{Ann}(\mathcal{R} h)[J]=\boldsymbol{M}^{r_{M}\left(h(b)^{*}\right)} .
$$

By the condition on $h, h(a)^{*}=h(b)^{*}$ since $a^{*}=b^{*}$; so $\mathcal{R} h$ is exoteric.

Proposition 3.10. Suppose L has a finite number of atoms, and denote by a the join of atoms of $L$. Let $h: L \rightarrow \downarrow a^{*}$ be the open quotient map. Then $\operatorname{Soc} \mathcal{R} L=$ ker $\mathcal{R} h$. Furthermore, $\mathcal{R} L / \operatorname{Soc} \mathcal{R} L \cong \mathcal{R}\left(\downarrow a^{*}\right)$.

Proof. For any $\alpha \in \mathcal{R} L, \alpha \in \operatorname{ker} \mathcal{R} h$ iff $h \circ \alpha=0$ iff $h(\operatorname{coz} \alpha)=0$ iff $\operatorname{coz} \alpha \wedge a^{*}=0$ iff $\operatorname{coz} \alpha \leq a$ iff $\operatorname{coz} \alpha$ is a join of atoms iff $\alpha \in \operatorname{Soc} \mathcal{R} L$. For the latter part, simply note that $\mathcal{R} h$ is onto as $h$ is a $C$-quotient map as observed earlier, and then invoke the first isomorphism theorem. 
REMARK 3.11. We stated in the introduction that a different proof of Proposition 3.5 is available. Here it is: By Brauer's proposition (see [12], Proposition 1 on page 62), a minimal ideal of $\mathcal{R} L$ is generated by an idempotent since $\mathcal{R} L$ is reduced. Now let $\eta$ be an idempotent such that $\langle\eta\rangle$ is a (nonzero) minimal ideal. We claim that $\operatorname{coz} \eta$ is an atom. Let $c$ be a cozero element of $L$ (say, $c=\operatorname{coz} \gamma$ ) such that $0<c \leq \operatorname{coz} \eta$. The minimality of $\langle\eta\rangle$ implies $\langle\gamma\rangle=\langle\eta\rangle$, so that $c=\operatorname{coz} \eta$. Hence, by complete regularity, if $x$ is any element of $L$ such that $0<x \leq \operatorname{coz} \eta$, then $x=\operatorname{coz} \eta$. So, for each $\varphi \in \operatorname{Soc} \mathcal{R} L, \operatorname{coz} \varphi$ is below a join of finitely many atoms, and is therefore a join of finitely many atoms. This establishes the inclusion $\subseteq$ in the proposition.

For the reverse inclusion, let $a$ be an atom of $L$, and (by the second of the results cited from [7] and stated in the paragraph preceding Proposition 3.8) pick an idempotent $\xi$ such that $a=\operatorname{coz} \xi$. For any $\alpha \in \mathcal{R} L$ such that $\alpha \xi \neq 0$, we have $0<\operatorname{coz} \alpha \xi \leq \operatorname{coz} \xi$, whence $\operatorname{coz} \xi \prec \prec \operatorname{coz} \xi=\operatorname{coz} \alpha \xi$. Thus, for some $\delta \in \mathcal{R} L, \xi=\delta \cdot \alpha \xi=\delta \xi \cdot \alpha \xi$, showing that $\langle\xi\rangle$ is a division ring, and hence, by Proposition 2 in [12] on page $63,\langle\xi\rangle$ is a minimal ideal. So if, for any $\varphi \in \mathcal{R} L, \operatorname{coz} \varphi$ is a join of finitely many atoms, then $\operatorname{coz} \varphi \prec \prec \operatorname{coz}\left(\zeta_{1}+\cdots+\zeta_{m}\right)$ for some idempotents $\zeta_{i}$ each generating a minimal ideal. Hence $\varphi$ is a multiple of $\zeta_{1}+\cdots+\zeta_{m}$, and is therefore in the socle.

\section{Contracting the socle.}

We observed in the previous section that a ring homomorphism induced by an onto frame homomorphism extends the socle to an ideal contained in the socle. What we aim to do here is identify certain types of frame homomorphisms for which the induced ring homomorphism contracts the socle to an ideal contained in the socle; that is, homomorphisms $h: L \rightarrow M$ for which $(\mathcal{R} h)^{-1}[\operatorname{Soc} \mathcal{R} M] \subseteq$ Soc $\mathcal{R} L$. We shall say a frame homomorphism is strong if it satisfies this property. In light of Corollary 3.6, surjective strong homomorphisms have the property that $(\mathcal{R} h)^{-1}[\operatorname{Soc} \mathcal{R} M]=\operatorname{Soc} \mathcal{R} L$.

We start by giving an example of a frame homomorphism which is not strong. Our example is spatial, so it should be read from the bottom up to keep in line with our frame-theoretic stance.

EXAmple 4.1. Endow $\mathbb{N} \cup\{0\}$ with the discrete topology, and let $X=Y=\mathbb{N} \cup\{0\}$. Let $h: X \rightarrow Y$ be the continuous function defined by $h(1)=1$ and $h(x)=0$ for all $1 \neq x \in X$. Next, let $g$ be the element of $C(Y)$ 
given by $g(y)=y$ for all $y \in Y$. Note that $g \notin \operatorname{Soc} C(Y)$. Let $\phi: C(Y) \rightarrow C(X)$ be the ring homomorphism $f \mapsto f \circ h$. We show that $g \in \phi^{-1}[\operatorname{Soc} C(X)]$. Indeed, if $x \neq 1$, then

$$
\phi(g)(x)=(g \circ h)(x)=g(h(x))=0,
$$

so that $\phi(g) \in \operatorname{Soc} C(X)$, and hence $g \in \phi^{-1}[\operatorname{Soc} C(X)]$. Thus $\phi^{-1}[\operatorname{Soc} C(X)] \nsubseteq$ $\nsubseteq \operatorname{Soc} C(Y)$.

It is well-known that the socle is invariant under an endomorphism. Since the homomorphism $\phi$ in Example 4.1 is, in fact, an endomorphism of $C(X)$, the example shows also that the socle is not necessarily "inverseinvariant" under endomorphisms.

Now we identify certain types of strong frame homomorphisms. Recall that every homomorphism $h: L \rightarrow M$ between completely regular frames lifts to the respective Stone-Čech compactifications as indicated in the following diagram:

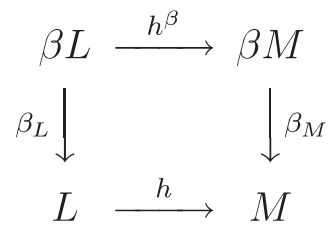

The map $h^{\beta}$ is given by $h^{\beta}(I)=\{x \in M \mid x \leq h(y)$ for some $y \in I\}$. A straightforward diagram-chasing argument shows that $h^{\beta}$ is dense if and only if $h$ is dense.

Definition 4.2. A frame homomorphism $h: L \rightarrow M$ is a $W$-map if, for all $c \in \operatorname{Coz} L, h^{\beta} r_{L}(c)=r_{M} h(c)$.

These homomorphisms are frame analogues of what Woods calls "WNmaps" in [15] defined for onto continuous functions $f: X \rightarrow Y$ by requiring that, for each cozero set $F$ of $Y, \operatorname{cl}_{\beta X} f^{-1}[F]=\left(f^{\beta}\right)^{-1}\left[\operatorname{cl}_{\beta Y} F\right]$, where $f^{\beta}$ is the Stone extension $f^{\beta}: \beta X \rightarrow \beta Y$ of $f$.

We will show that dense W-maps are strong. If $\mathcal{R} h$ were an isomorphism for every dense W-map $h$, then of course the result would be trivial. So we start by giving an example of a dense W-map for which $\mathcal{R} h$ is not an isomorphism. Our example will be facilitated by the following characterization of $\mathrm{W}$-maps.

LEMma 4.3. A frame homomorphism $h: L \rightarrow M$ is a W-map iff for every $c \in \operatorname{Coz} L$ and $y \in M, y \prec \prec h(c)$ implies $y \leq h(s)$ for some $s \prec \prec$. 
Proof. Let $h$ be a W-map, $y$ be an element of $M$ and $c$ a cozero element of $L$ such that $y \prec \prec h(c)$. Then $y \in r_{M} h(c)=h^{\beta} r_{L}(c)$. Therefore $y \leq h(s)$ for some $s \in r_{L}(c)$.

Conversely, observe first that, for any $c \in \operatorname{Coz} L$, in fact, for any $c \in L$, the inclusion $h^{\beta} r_{L}(c) \subseteq r_{M} h(c)$ always holds since, for any $s \in L, s \prec \prec c$ implies $h(s) \prec \prec h(c)$. For the other inclusion, let $y \in r_{M} h(c)$. Then $y \prec \prec h(c)$, and so, by hypothesis, $y \leq h(s)$ for some $s \in r_{L}(c)$, that is, $y \in h^{\beta} r_{L}(c)$. This establishes the desired inclusion.

Some more background. The Booleanization of a frame $L$ is the Boolean frame $\mathfrak{B} L$ whose underlying set is $\mathfrak{B} L=\left\{x^{* *} \mid x \in L\right\}$ with meet as in $L$ and join $(\bigvee S)^{* *}$ for each $S \subseteq \mathfrak{B} L$. The map $L \rightarrow \mathfrak{B} L$ which sends each $x \in L$ to $x^{* *}$ is a dense onto frame homomorphism. We denote it by $b$.

Our example will be based on special types of frames. The reader will recall that a $P$-frame is a frame each of whose cozero elements is complemented, and that a frame $L$ is extremally disconnected (or strongly projectable) if $x^{*} \vee x^{* *}=1$ for each $x \in L$. See [8] for several characterizations of $P$-frames. However, we shall need the following characterization (which does not appear in [8]) in terms of W-maps.

Proposition 4.4. The following are equivalent for a completely regular frame $L$ :

(1) $L$ is a P-frame.

(2) Every frame homomorphism $h: L \rightarrow M$ is a W-map.

(3) b: $L \rightarrow \mathfrak{B} L$ is a $W$-map.

Proof. $\quad(1) \Rightarrow$ (2): Let $c \in \operatorname{Coz} L$ and $y \in M$ such that $y \prec \prec h(c)$. Since $y \leq h(c)$ and $c \prec \prec c$, as $L$ is a $P$-frame, it follows from Lemma 4.3 that $h$ is a W-map.

$(2) \Rightarrow(3)$ : Trivial

(3) $\Rightarrow(1)$ : Let $c \in \operatorname{Coz} L$. To avoid ambiguity, write $\preceq \preceq$ for the completely below relation in $\mathfrak{B} L$. Now, $b(c) \preceq \preceq b(c)$ implies there exists $d \prec \prec c$ in $L$ such that $b(c) \leq b(d)$, by Lemma 4.3. Notice that $b(c) \leq b(d)$ means $c^{* *} \leq d^{* *}$, and $d \prec \prec c$ implies $d^{* *} \prec c$. Therefore $c \leq c^{* *} \leq d^{* *} \prec c$, which implies $c$ is complemented.

Example 4.5. Let $X$ be a $P$-space which is not extremally disconnected. Such a space does exist (see [10], $4 \mathrm{~N})$. Let $L=\subseteq X$. Then $L$ is a $P$ frame which is not extremally disconnected. We claim that b: $L \rightarrow \mathfrak{B} L$ is 
not coz-onto. Suppose, by way of contradiction, that $b$ is coz-onto. Let $x \in L$. Then $x^{* *} \in \operatorname{Coz} \mathfrak{B} L$, since $\mathfrak{B} L$ is Boolean. Therefore there exists $c \in \operatorname{Coz} L$ such that $b(c)=x^{* *}$, that is, $c^{* *}=x^{* *}$. Since $c^{* *}=c$ and $c$ is complemented, it follows that $x^{* *} \vee x^{*}=1$. But this makes $L$ extremally disconnected since $x$ is arbitrary. It follows therefore that $b$ is not a $C$-quotient map, and consequently $\mathcal{R} b$ is not an isomorphism. Thus, $b$ is a dense (onto) W-map for which the induced ring homomorphism is not an isomorphism.

Now we show that if $h$ is a dense W-map, then $\mathcal{R} h$ contracts the socle to an ideal contained in the socle.

Proposition 4.6. A dense $W$-map is strong.

Proof. Let $h: L \rightarrow M$ be a dense W-map. Let $\alpha \in(\mathcal{R} h)^{-1}[\operatorname{Soc} \mathcal{R} M]$. Then $h \circ \alpha \in \operatorname{Soc} \mathcal{R} M$. We shall use subscripts to indicate where an $\boldsymbol{M}$-ideal resides. Take finitely many points $I_{1}, \ldots, I_{k}$ of $\beta M$ such that

$$
\operatorname{Ann}(h \circ \alpha)=\boldsymbol{M}_{M}^{I_{1}} \cap \cdots \cap \boldsymbol{M}_{M}^{I_{k}} \text {. }
$$

For each $i \in\{1, \ldots, k\}, h_{*}^{\beta}\left(I_{i}\right)$ is a point of $\beta L$. We claim that

$$
\operatorname{Ann} \alpha=\boldsymbol{M}_{L}^{h_{*}^{\beta}\left(I_{1}\right)} \cap \cdots \cap \boldsymbol{M}_{L}^{h_{*}^{\beta}\left(I_{k}\right)} .
$$

Let $\varphi \in$ Ann $\alpha$. Then

$$
(h \circ \varphi) \cdot(h \circ \alpha)=(\mathcal{R} h)(\varphi) \cdot(\mathcal{R} h)(\alpha)=(\mathcal{R} h)(\varphi \alpha)=\mathbf{0},
$$

and hence $h \circ \varphi \in \operatorname{Ann}(h \circ \alpha)$. Therefore $r_{M}(\operatorname{coz}(h \circ \varphi)) \subseteq I_{i}$, for each $i \in\{1, \ldots, k\}$. But $\operatorname{coz}(h \circ \varphi)=h(\operatorname{coz} \varphi)$; so, for each $i$ we have $r_{M}(h(\operatorname{coz} \varphi)) \subseteq I_{i}$. Thus, $h^{\beta} r_{L}(\operatorname{coz} \varphi) \subseteq I_{i}$ for each $i$ since $h^{\beta} r_{L} \leq r_{M} h$ always. Consequently, $r_{L}(\operatorname{coz} \varphi) \subseteq h_{*}^{\beta}\left(I_{i}\right)$, that is, $\varphi \in M_{L}^{h_{*}^{\beta}\left(I_{i}\right)}$ for each $i$. Therefore

$$
\text { Ann } \alpha \subseteq \boldsymbol{M}_{L}^{h_{*}^{\beta}\left(I_{1}\right)} \cap \cdots \cap \boldsymbol{M}_{L}^{h_{*}^{\beta}\left(I_{k}\right)} .
$$

To show the other inclusion, let $\tau \in M_{L}^{h_{*}^{\beta}\left(I_{i}\right)}$, for each $i \in\{1, \ldots, k\}$. Then $r_{L}(\operatorname{coz} \tau) \subseteq h_{*}^{\beta}\left(I_{i}\right)$, and hence $h^{\beta} r_{L}(\operatorname{coz} \tau) \subseteq h^{\beta} h_{*}^{\beta}\left(I_{i}\right) \subseteq I_{i}$. Since $h$ is a W-map, this implies

$$
r_{M}(h(\operatorname{coz} \tau)) \subseteq I_{i},
$$

that is, $r_{M}(\operatorname{coz}(h \circ \tau)) \subseteq I_{i}$, so that $h \circ \tau \in \boldsymbol{M}_{M}^{I_{i}}$. As this holds for each $i$, we deduce that $h \circ \tau \in$ Ann $(h \circ \alpha)$. Consequently,

$0=\operatorname{coz}((h \circ \tau) \cdot(h \circ \alpha))=\operatorname{coz}(h \circ \tau) \wedge \operatorname{coz}(h \circ \alpha)=h(\operatorname{coz} \tau \wedge \operatorname{coz} \alpha)=h(\operatorname{coz} \tau \alpha)$, 
which implies $\operatorname{coz} \tau \alpha=0$ by density. Thus, $\tau \alpha=\mathbf{0}$, and hence the reverse inclusion holds.

In light of Corollary 3.6, we have the following:

Corollary 4.7. If $h: L \rightarrow M$ is a dense onto W-map, then $(\mathcal{R} h)^{-1}[\operatorname{Soc} \mathcal{R} M]=\operatorname{Soc} \mathcal{R} L$.

Lest one conjecture falsely, we give an example of a dense onto frame homomorphism $h: L \rightarrow M$ for which $(\mathcal{R} h)^{-1}[\operatorname{Soc} \mathcal{R} M]=\operatorname{Soc} \mathcal{R} L$, but $h$ is not a W-map.

EXAMPLE 4.8. Let $L=\mathfrak{O R}$ and consider the map $b: L \rightarrow \mathfrak{B} L$. Neither $L$ nor $\mathfrak{B} L$ has atoms, so each of the rings $\mathcal{R} L$ and $\mathcal{R}(\mathfrak{B} L)$ has zero socle. Since $b$ is dense, $\mathcal{R} b$ is one-to-one (see [4], Lemma 2). It follows therefore that $(\mathcal{R} b)^{-1}[\operatorname{Soc} \mathcal{R}(\mathfrak{B} L)]=\{\boldsymbol{0}\}=\operatorname{Soc} \mathcal{R} L$. However, by Proposition 4.4, $b$ is not a W-map.

A closer look at the proof of Proposition 4.6 shows that what makes it go through, apart from the density of $h$, is the fact that $\mathcal{R} h$ contracts any maximal ideal to a maximal ideal. As it turns out, this property actually characterizes W-maps as we now show.

Proposition 4.9. A homomorphism $h: L \rightarrow M$ is a $W$-map iff $(\mathcal{R} h)^{-1}\left[\boldsymbol{M}_{M}^{I}\right]=\boldsymbol{M}_{L}^{h_{*}^{\beta}(I)}$ for each point I of $\beta M$.

Proof. $(\Leftarrow)$ : Let $c \in \operatorname{Coz} L$. Since we always have $h^{\beta} r_{L}(c) \subseteq r_{M} h(c)$, we need to show that $r_{M} h(c) \subseteq h^{\beta} r_{L}(c)$. If $h^{\beta} r_{L}(c)=1_{\beta M}$, there is nothing to prove. So suppose $h^{\beta} r_{L}(c) \neq 1_{\beta M}$ and let $I$ be a point of $\beta M$ such that $h^{\beta} r_{L}(c) \subseteq I$. Take $\gamma \in \mathcal{R} L$ such that $c=\operatorname{coz} \gamma$. Then $r_{L}(\operatorname{coz} \gamma) \subseteq h_{*}^{\beta}(I)$, which implies $\gamma \in \boldsymbol{M}_{L}^{h_{*}^{\beta}(I)}$. So, by hypothesis, $\gamma \in(\mathcal{R} h)^{-1}\left[\boldsymbol{M}_{M}^{I}\right]$, that is, $(\mathcal{R} h)(\gamma)=$ $=h \circ \gamma \in \boldsymbol{M}_{M}^{I}$. Thus,

$$
I \supseteq r_{M}(\operatorname{coz}(h \circ \gamma))=r_{M}(h(\operatorname{coz} \gamma))=r_{M} h(c) .
$$

Now, since every element of $\beta M$ is the meet of points above it, it follows that

$$
\begin{gathered}
r_{M} h(c) \leq \bigwedge\left\{J \in \beta M \mid J \text { is a point above } h^{\beta} r_{L}(c)\right\}=h^{\beta} r_{L}(c) . \\
(\Rightarrow) \text { : Let } \alpha \in(\mathcal{R} h)^{-1}\left[\boldsymbol{M}_{M}^{I}\right] \text {. Then } h \circ \alpha \in \boldsymbol{M}_{M}^{I}, \text { and hence } \\
r_{M}(\operatorname{coz}(h \circ \alpha))=r_{M}(h(\operatorname{coz} \alpha))=h^{\beta} r_{L}(\operatorname{coz} \alpha) \subseteq I .
\end{gathered}
$$


Thus, $r_{L}(\operatorname{coz} \alpha) \subseteq h_{*}^{\beta}(I)$. That is, $\alpha \in \boldsymbol{M}_{L}^{h_{*}^{\beta}(I)}$, and therefore $(\mathcal{R} h)^{-1}\left[\boldsymbol{M}_{M}^{I}\right] \subseteq$ $\subseteq \boldsymbol{M}_{L}^{h_{*}^{\beta}(I)}$. For the reverse inclusion, let $\varphi \in \boldsymbol{M}_{L}^{h_{*}^{\beta}(I)}$. Therefore $r_{L}(\operatorname{coz} \varphi) \subseteq$ $\subseteq h_{*}^{\beta}(I)$, which implies

$$
h^{\beta} r_{L}(\operatorname{coz} \varphi) \subseteq h^{\beta} h_{*}^{\beta}(I) \subseteq I,
$$

and consequently, by hypothesis,

$$
r_{M}(\operatorname{coz}(h \circ \varphi))=r_{M} h(\operatorname{coz} \varphi) \subseteq I .
$$

Therefore $h \circ \varphi \in \boldsymbol{M}_{M}^{I}$; that is, $(\mathcal{R} h)(\varphi) \in \boldsymbol{M}_{M}^{I}$, implying $\varphi \in(\mathcal{R} h)^{-1}\left[\boldsymbol{M}_{M}^{I}\right]$. This proves the reverse inclusion.

Remark 4.10. We proved Proposition 4.6 directly. We could, after observing the last proposition, have deduced it as a corollary of the following ring-theoretic observation: If $\phi: A \rightarrow B$ is a one-to-one ring homomorphism such that $\phi^{-1}[M]$ is a maximal ideal of $A$ for each maximal ideal $M$ of $B$, then $\phi^{-1}[\operatorname{Soc} B] \subseteq \operatorname{Soc} A$. To see this, let $a \in \phi^{-1}[\operatorname{Soc} B]$, and select maximal ideals $M_{1}, \ldots, M_{k}$ of $B$ such that Ann $\phi(a)=M_{1} \cap \cdots \cap M_{k}$. One checks easily, using the fact that $\phi$ is one-to-one, that Ann $a=$ $=\phi^{-1}\left[M_{1}\right] \cap \cdots \cap \phi^{-1}\left[M_{k}\right]$, so that $a \in \operatorname{Soc} A$.

W conclude with the following comment. If we strengthen the definition of W-map and define an $N$-map to be a homomorphism $h: L \rightarrow M$ such that $h^{\beta} r_{L}(a)=r_{M} h(a)$ for every $a \in L$ (instead of just the cozero elements), then it turns out that $\mathcal{R} h$ is an isomorphism whenever $h$ is a dense onto N-map. To show this, we first demonstrate that $h^{\beta}$ is onto. Given $a \in M$ take $b \in L$ such that $h(b)=a$. Then $h^{\beta}\left(r_{L}(b)\right)=r_{M} h(b)=$ $=r_{M}(a)$. Since the elements $r_{M}(x)$, for $x \in M$, generate $\beta M$, it follows that $h^{\beta}$ is onto. As remarked earlier, the density of $h$ implies that of $h^{\beta}$. So, in fact, $h^{\beta}$ is an isomorphism since it is also one-to-one as any dense frame homomorphism with regular domain and compact codomain is one-to-one. Now we argue from this that $h$ is coz-onto. Let $c \in \mathrm{Coz} M$. Since $\beta M \rightarrow M$ is coz-onto ([5], Corollary 5), there exists $J \in \operatorname{Coz} \beta M$ such that $\bigvee J=c$. Since $h^{\beta}$ is coz-onto (as it is an isomorphism), there exists $I \in \operatorname{Coz} \beta L$ such that $h^{\beta}(I)=J$. Then $\bigvee I$ is a cozero element of $L$ mapped to $c$ by $h$. Finally, we show that $h$ is almost coz-codense. So, suppose $h(c)=1$ for some $c \in \operatorname{Coz} L$. Then, in view of $h$ being an N-map, $h^{\beta} r_{L}(c)=r_{M} h(c)=1_{\beta M}$, which implies $r_{L}(c)=1_{\beta L}$ since $h^{\beta}$ is one-to-one, and hence $c=1$. Thus, $h$ is a $C$-quotient map, and hence $\mathcal{R} h$ is an isomorphism. 
A similar argument, taking into account the fact that the elements $r_{M}(c)$, for $c \in \operatorname{Coz} L$, generate $\beta M$, shows that if $h$ is a coz-onto dense Wmap, then $\mathcal{R} h$ is an isomorphism.

Acknowledgments. I thank the referee most heartily for helpful remarks and observations that have strengthened the paper.

\section{REFERENCES}

[1] R. N. BaLl - J. Walters-Wayland, $C$ - and $C^{*}$-quotients in pointfree topology, Dissertationes Mathematicae (Rozprawy Mat.), Vol. 412 (2002), p. 62.

[2] B. Banaschewski, The real numbers in pointfree topology, Textos de Matemática Série B, No. 12, Departamento de Matemática da Universidade de Coimbra, 1997.

[3] B. BANASCHEWSKI, Gelfand and exchange rings: Their spectra in pointfree topology, The Arabian J. Science and Engineering, 25 (2000), pp. 3-22.

[4] B. Banaschewski, On the function ring functor in pointfree topology, Appl. Categor. Struct., 13 (2005), pp. 305-328.

[5] B. Banaschewski - C. Gilmour, Pseudocompactness and the cozero part of a frame, Comment. Math. Univ. Carolinae, 37(3) (1996), pp. 577-587.

[6] T. Dube, Some ring-theoretic properties of almost P-frames, Alg. Univ., 60 (2009), pp. 145-162.

[7] T. DuBE, Notes on pointfree disconnectivity with a ring-theoretic slant, Appl. Categor. Struct., 18 (2010), pp. 55-72.

[8] T. DuBE, Concerning P-, essential $P$ - and strongly zero-dimensional frames, Alg. Univ., 69 (2009), pp. 115-138.

[9] M. W. Evans, Exoteric homomorphisms, J. Pure Appl. Alg., 49 (1987), pp. $117-131$.

[10] L. Gillman - M. Jerison, Rings of Continuous Functions, Van Nostrand, Princeton, 1960.

[11] M. Henriksen - M. Jerison, The space of minimal prime ideals of a commutative ring, Trans. Amer. Math. Soc., 115 (1965), pp. 110-130.

[12] J. LambeK, Lecture notes on rings and modules, Chelsea Publ. Co. (New York, 1976).

[13] P. T. Johnstone, Stone Spaces, Cambridge Univ. Press (Cambridge, 1982).

[14] O. A. S. KARAMZADEH - M. Rostami, On the intrisic topology and some related ideals of $C(X)$, Proc. Amer. Math. Soc., 93 (1) (1985), pp. 179-184.

[15] R. G. Woods, Maps that characterize normality properties and pseudocompactness, J. London Math. Soc., 7 (2) (1973), pp. 453-461.

Manoscritto pervenuto in redazione il 22 settembre 2008. 
